Accepted author's manuscript. Published in final edited form as: Gut 2020 (in press).

Publisher DOI: 10.1136/gutjnl-2020-322237

\title{
Risk of digestive cancers in a cohort of 69,460 5-year survivors of childhood cancer in Europe: The PanCareSurFup study
}

Raoul C Reulen, PhD (1), Kwok F Wong, PhD (1), Chloe J Bright, PhD (1), David L Winter, HNC (1), Daniela Alessi, PhD (2), Rodrigue S Allodji, PhD (3), Francesca Bagnasco, PhD (4), Edit Bárdi, MD (5, 6), Andrea Bautz, PhD (7), Julianne Byrne, PhD (8), Elizabeth AM Feijen, PhD (9), Fidler-Benaoudia M Miranda, PhD (10), Ibrahim Diallo, PhD (11), Stanislaw Garwicz, MD, PhD (12), Desiree Grabow, PhD (13), Thorgerdur Gudmundsdottir, MD (7, 14), Joyeeta Guha, PhD (1), Nadia Haddy, PhD (11), Stine Høgsholt, PhD (7, 15), Moncilo Jankovic, MD (16), Peter Kaatsch, PhD (17), Melanie Kaiser, $\mathrm{PhD}$ (13), Rahel Kuonen, MSc (18), Helena Linge, PhD (19), Hilde Øfstaas, PhD (20), Cecile M Ronckers, PhD (9, 21), EvaMaria Hau, MD (18, 22), Roderick Skinner, MD, PhD (23), Flora E van Leeuwen, PhD (24), Jop C Teepen, PhD (25), Cristina Veres, MSc (11), Wael Zrafi, MD (11), Ghazi Debiche, MD (11), Damien Llanas, MSc (11), Monica Terenziani, MD (26), Giao Vu-Bezin, PhD (11), Finn Wesenberg, MD, PhD (27), Thomas Wiebe, MD (19), Carlotta Sacerdote, PhD (2), Zsuzsanna Jakab , MD (28), Riccardo Haupt, MD (29), Päivi M Lähteenmäki, MD (30), Lorna Zadravec Zaletel, MD (31), Claudia E Kuehni, PhD (18, 22), Jeanette Winther, PhD (7, 32), Florent de Vathaire, PhD (11), Leontien C Kremer, MD, PhD $(9,21)$, Lars Hjorth, MD, PhD (12), Michael M Hawkins, DPhil (1)

1) Centre for Childhood Cancer Survivor Studies, Institute of Applied Health Research, Robert Aitken Building, University of Birmingham, Birmingham, UK 2) Childhood Cancer Registry of Piedmont, Cancer Epidemiology Unit, Department of Medical Sciences, University of Turin and AOU Città della Salute e della Scienza di Torino, Italy

3) Cancer and Radiation Team, Center for Research in Epidemiology and Population Health, INSERM U1018, University Paris Saclay, Gustave Roussy, Villejuif, France

4) Epidemiology and Biostatistics Unit, Instituto Giannina Gaslini, Genova, Italy

5) 2nd Department of Pediatrics, Semmelweis University, Budapest, Hungary

6) Kepler Universitätsklinikum, Linz, Austria

7) Danish Cancer Society Research Center, Childhood Cancer Research Group, Copenhagen, Denmark

8) Boyne Research Institute, 5 Bolton Square, East, Drogheda, A92 RY6K, Ireland

9) Princess Maxima Center for Pediatric Oncology, Utrecht, The Netherlands

10) Epidemiology and Prevention Research, Alberta Health Services, Calgary, AB, Canada, Departments of Oncology and Community Health Sciences, University of Calgary, Calgary, AB, Canada

11) Radiation Epidemiology Team, Center for Research in Epidemiology and Population Health, INSERM U1018, University Paris Saclay, Gustave Roussy, Villejuif, France

12) Department of Clinical Sciences, Skåne University Hospital, Lund, Sweden

13) German Childhood Cancer Registry, University Medical Centre Mainz, Germany

14) Children's Hospital, Landspitali University Hospital, Reykjavik, Iceland

15) Department of Pediatrics, Aarhus University Hospital, Aarhus, Denmark

16) Pediatric Clinic, University of Milano-Bicocca, Hospital San Gerardo, Via Donizetti 33, Monza-Italy

17) German Childhood Cancer Registry, Institute for Medical Biostatistics, Epidemiology and Informatics, University Medical Center, Johannes Gutenberg University Mainz, Germany

18) Swiss Childhood Cancer Registry, Institute of Social and Preventive Medicine, University of Bern, Bern, Switzerland

19) Lund University, Skane University Hospital, Department of Clinical Sciences, Paediatrics, Lund, Sweden

20) Norwegian National Advisory Unit on solid tumors in children, Norway

21) Department of pediatric Oncology, Emma Children's Hospital \& Academic Medical Center, Amsterdam

22) Department of Paediatrics, University Children's Hospital of Bern, University of Bern, Switzerland

23) Great North Children's Hospital, Newcastle upon Tyne Hospitals NHS Foundation Trust, and Newcastle University Centre for Cancer,, Newcastle University, Newcastle upon Tyne, UK

24) Department of Epidemiology, Division of Psychosocial Research \& Epidemiology, Netherlands Cancer Institute, Amsterdam, The Netherlands

25) Princess Maxima Center for Pediatric Oncology, Utrecht, The Netherlands.

26) Pediatric Oncology Unit, Fondazione IRCCS Istituto Nazionale dei Tumori , Milano, Italy

27) Norwegian Cancer Registry and Dept. of Pediatric Medicine, Oslo University Hospital and Institute of Clinical Medicine, Faculty of medicine, University of Oslo, Norway

28) Hungarian Childhood Cancer Registry, 2nd Department of Pediatrics, Semmelweis University, Budapest, Hungary

29) Epidemiology and Biostatistics Unit, IRCCS Istituto Giannina Gaslini, Genova, Italy

30) Turku University and Turku University Hospital, Department of Pediatrics and Adolescent Medicine, Turku, Finland

31) Division of Radiotherapy, Institute of Oncology, Ljubliana, Slovenia

32) Department of Clinical Medicine, Faculty of Health, Aarhus University and University Hospital, Aarhus, Denmark

tDeceased 27th November 2018, to whom this work is dedicated.

Word count: 3740

Abbreviations: AER: absolute excess risks; Cl: confidence interval; HL: Hodgkin lymphoma; ICCC :International Classification of Childhood Cancers; ICD: International 
Classification of Diseases; ICD-O-3: International Classification of Diseases for Oncology; RER: relative excess risk; RR: relative risk; SIR: standardised incidence ratio; SPN: subsequent primary neoplasm; STS: soft-tissue sarcoma; WT: Wims tumour.

Corresponding author: Mike M Hawkins, Centre for Childhood Cancer Survivor Studies, Institute of Applied Health Research, Robert Aitken Building, University of Birmingham, Birmingham, B15 2TT; email: m.m.hawkins@bham.ac.uk, telephone: +44(0)1214147924 


\section{Abstract}

Background: Survivors of childhood cancer are at risk of subsequent primary neoplasms (SPNs), but the risk of developing specific digestive SPNs beyond age 40 years remains uncertain. We investigated risks of specific digestive SPNs within the largest available cohort worldwide.

Methods: The PanCareSurFup cohort includes 69,460 five-year survivors of childhood cancer from 12 countries in Europe. Risks of digestive SPNs were quantified using standardised incidence ratios (SIRs), absolute excess risks, and cumulative incidence. Results: 427 digestive SPNs (214 colorectal, 62 liver, 48 stomach, 44 pancreas, 59 other) were diagnosed in 413 survivors. Wims tumour (WT) and Hodgkin lymphoma (HL) survivors were at greatest risk ( $\mathrm{SIR}=12.1 ; 95 \% \mathrm{Cl}: 9.6-15.1 ; \mathrm{SIR}=7.3 ; 95 \% \mathrm{Cl}: 5.9-9.0$, respectively). The cumulative incidence increased the most steeply with increasing age for WT survivors, reaching $7.4 \%$ by age 55 and $9.6 \%$ by age 60 years $(1.0 \%$ expected based on general population rates). Regarding colorectal SPNs, WT and HL survivors were at greatest risk; both 7 -times that expected. By age 55 years, $2.3 \%$ of both WT $(95 \% \mathrm{Cl}: 1.4-3.9)$ and $\mathrm{HL}$ (95\% Cl:1.6-3.2) survivors had developed a colorectal SPN-comparable to the risk among members of the general population with at least 2 first-degree relatives affected.

Conclusions: Colonoscopy surveillance before age 55 is recommended in many European countries for individuals with a family history of colorectal cancer, but not for WT and HL survivors despite a comparable risk profile. Clinically, serious consideration should be given to the implementation of colonoscopy surveillance whilst further evaluation of its benefits, harms and cost-effectiveness in WT and HL survivors is undertaken. 


\section{What is already known about this subject?}

Survivors of childhood cancer are at risk of subsequent primary neoplasms (SPNs) including digestive SPNs. Increasing number of survivors are now reaching their forties and beyond, but the risk of developing specific digestive SPNs, such colorectal, liver, stomach and pancreas, beyond age 40 years remains uncertain.

\section{What are the new findings?}

The cumulative incidence of developing any digestive SPN for WT survivors reaches $7.4 \%$ by age 55 years and $9.6 \%$ by age 60 years. The cumulative incidence for colorectal SPNs for both WT and HL survivors is $2.3 \%$ by age 55 ; which is comparable to the risk among members of the general population with at least 2 first-degree relatives affected by colorectal cancer and for whom in many countries colonoscopy surveillance is recommended.

\section{How might it impact on clinical practice in the foreseeable future?}

This study provides evidence that should aid the development of international guidelines for surveillance of colorectal cancer among survivors of childhood cancer. Although implementation of colorectal cancer surveillance would require evaluation of its benefits, harms and cost-effectiveness, the high risks identified in this study suggest that, if not already, in most countries colonoscopy surveillance among childhood WT and HL survivors should be seriously considered. 


\section{Introduction}

Over the last few decades five-year survival after childhood cancer has increased substantially and is currently over $80 \%$ [1]. However, survivors of childhood cancer are at risk of long-term adverse health conditions, including subsequent primary neoplasms (SPNs) [2-5]. Although the median age of survivors is currently around thirty, an increasing number of survivors are now reaching their forties and beyond. Hence it is important to investigate among survivors the excess risks of cancers that occur most frequently beyond age 40 years in the general population, such as cancers of the digestive system [6]. Survivors of childhood cancer may be at excess risk of developing such digestive SPNs due to previous radiotherapy given involving the abdomen or treatment with specific chemotherapeutic agents [7-9]. Previous work has shown that digestive cancers contribute nearly $20 \%$ to the total excess risk of SPNs observed beyond age 40 in survivors of childhood cancer [3]. Even a small increased relative risk compared to the general population sustained into old age could lead to a considerable excess numbers of survivors developing a digestive SPN. Quantification of the risks would aid in identifying those survivors at highest risks and in estimating the number of digestive SPNs that could potentially be prevented, through for example surveillance. Clearly, there is a need for large scale studies to quantify the risks of digestive SPNs, however, to our knowledge, most studies conducted thus far lacked statistical power to satisfactorily address the very long-term risks of digestive SPNs [2-4,711]. For example, the largest cohort study to date not included in the current study included 14,358 5-year survivors of childhood cancer and 45 digestive SPNs, of which 14 occurred beyond age 40 years [7].

The principal objective of this largest-ever cohort study was to investigate the risks of developing SPNs in specific sites of the digestive system-including colorectal, liver, stomach, and pancreas SPNs-among 5-year survivors of childhood cancer beyond age 40 years. The current study used data from a large-scale Pan-European cohort of nearly 70,000 5-year survivors of childhood cancer with 427 observed digestive SPNs, of which 230 occurred beyond age 40 years. 


\section{Methods}

PanCare Childhood and Adolescent Cancer Survivor Care and Follow-Up Studies (PanCareSurFup)

PanCareSurFup is a consortium of 16 European institutions in 12 countries established in February 2011 and funded by the 7th Framework Programme of the European Commission (www.pancaresurfup.eu) $[12,13]$. The overall aims of the PanCareSurFup consortium are: (i) to conduct studies into long-term complications of treatment for cancer, (ii) to establish guidelines for clinical follow-up of survivors, (iii) and to disseminate the results and provide training and workshops for stakeholders. PanCareSurFup is the largest ever collaborative study undertaken to comprehensively investigate specific long-term adverse health outcomes among survivors of childhood and adolescent cancer [14]. One of the principal objectives of PanCareSurFup was to estimate the risks of developing SPNs among five-year survivors of a cancer diagnosed under age 20 years [15,16]. A pooled cohort of survivors of childhood cancer was established using data from 13 different sub-cohorts-ascertained from cancer registries or major treatment centres-across 12 countries within Europe, including: Great Britain, Norway, Sweden, Finland, Denmark, Iceland, France, Switzerland, Hungary, Slovenia, Italy, and the Netherlands (eTable 1). Ethical approval was not obtained specifically for this study as it involved pooling of non-identifiable data. Ethical approval was obtained within the country of origin of each contributing sub-cohort separately.

\section{Childhood cancer classification}

Site and type of childhood tumour were coded using a variety of different tumour classification systems across the sub-cohorts provided by the countries; however, for the pooled cohort all tumour classification codes were converted into the third revision of the International Classification of Diseases for Oncology (ICD-O-3) using the Cancer Registry Tools program developed by the International Agency for Research on Cancer and the International Association of Cancer Registries [17]. Individuals were excluded from the pooled cohort if: a) the first primary diagnosis was myelodysplastic syndrome, Langerhans cell histiocytosis, a chronic myeloproliferative or lymphoproliferative disorder or a immunoproliferative disease; b) tumour codes were of a non-malignant behaviour code except for intra-cranial and bladder tumours and survivors; $c$ ) tumour codes were not classifiable according to the International Classification of Childhood Cancers (ICCC) (3 ${ }^{\text {rd }}$ revision)[18] (eFigure 1).

\section{Subsequent primary neoplasm ascertainment}

SPNs were ascertained through: linkage with population-based national cancer registries, 
follow-up clinics, questionnaires, available medical records, linkage with national mortality registries, and linkage with health insurance registries (eTable 1). SPNs were classified by digestive site using the International Classification of Diseases (ICD) (eTable 2). Potential digestive SPNs were only included if the behaviour code was malignant (ICD-O behaviour code: 3). SPNs were validated principally using pathology reports and occasionally other definitive diagnostic reports.

\section{Statistical Analysis}

The period at risk of developing a SPN commenced five years from childhood cancer diagnosis until the first occurrence of: death, loss-to-follow-up, or study exit date. Survivors lost to follow-up were censored at last known date alive. Multiple SPNs per survivor were permitted in all analyses unless otherwise specified to avoid bias because the general population cancer registration rates include multiple primaries. Standardised incidence ratios (SIR) were calculated as the ratio of observed to expected number of digestive neoplasms [19]. Expected numbers were estimated by accumulating person-years at risk within country, sex, age, and calendar year specific strata and multiplying by the corresponding digestive neoplasms incidence rates in the general population. General population incidence rates for each specific digestive neoplasm site by country, sex, age (5-year bands), and calendar year (1-year bands) were obtained from the Cancer Incidence in Five Continents and the European Cancer Observatory database [20,21]. Since cancer site-specific neoplasm rates do not exist for Hungary these were estimated by dividing age, sex and calendar year specific cancer incidence rates from the neighbouring country Slovakia by corresponding Slovakian mortality rates and then multiplying these by available Hungarian mortality rates [22]. Absolute excess risks (AERs) were calculated as the observed minus expected number of specific digestive neoplasms, divided by the number of person-years at risk, multiplied by 100,000. Multivariable Poisson regression models were fitted to estimate the relative risk $(R R)$ and the relative excess risk (RER) of each digestive SPN taking into account the simultaneous effect of the factors: sex, type of childhood cancer, country, age at diagnosis, era of diagnosis, and attained age [23]. Relative risks (RR) may be interpreted as the ratio of SIRs, adjusted for relevant co-factors fitted. Similarly, relative excess risks (RER) may be interpreted as the ratio of AERs, adjusted for relevant co-factors fitted. Negative binomial regression was used when the Poisson regression model fit showed signs of overdispersion [24].

Cumulative incidence of the first digestive SPN was estimated by treating death as a competing risk $[25,26]$ and compared to the expected cumulative incidence derived from general population incidence rates by the conditional (Ederer II) method [27]. For colorectal cancer, the cumulative incidence in survivors was also compared to the expected cumulative 
incidence from subgroups of the general population known to have an increased risk of colorectal cancer and for whom undergoing colonoscopy surveillance from an early age is recommended in many countries; $i$ ) those with at least 2 first-degree relatives affected by colorectal cancer and ii) those with at least one first-degree relative affected. These expected incidences were estimated using age-specific RRs of colorectal cancer for these subgroups compared to the general population from published meta-analyses and multiplying the age-specific RRs to the corresponding age-specific colorectal cancer incidence rates from the relevant national general population $[28,29]$.

Tests for linear trend for a specific factor (e.g. attained age) were performed by using a likelihood-ratio test comparing the deviance of a model containing the factor of interest as a continuous variable (e.g. 1,2,3,4) to the deviance of a model without this factor. Statistical significance for all analyses was defined as a two-sided P-value of less than 0.05. All analyses were carried out in Stata 16 (StataCorp, College Station, Texas).

\section{Patient and Public Involvement}

The PanCare network is a multidisciplinary pan-European network of professionals, survivors and their families. Members of PanCare meet biannually and the work in this manuscript has been presented at these meetings and has benefited from feedback from survivors and their families. 


\section{Results}

\section{Cohort Characteristics}

Following five-year survival from childhood cancer diagnosis, 69,460 survivors in the pooled cohort were followed up for 1,264,624 person-years with a mean and median follow-up of 18.2 and 16.3 years, respectively (range: 5-70 years). Loss-to-follow-up did not exceed 6\% (eTable 1). Over the follow-up period, 427 digestive SPNs were diagnosed in 413 survivors with 12 survivors diagnosed with two digestive SPNs and one survivor with three digestive SPNs. Digestive SPNs were most frequently diagnosed after Hodgkin's lymphoma (HL) $(\mathrm{N}=85)$ and Wilms tumour (WT) $(\mathrm{N}=77)$ (Table 1). From the 427 digestive SPNs, 214 (50.1\%) were colorectal, $62(14.5 \%)$ liver, 48 (11.2\%) stomach, 44 (10.3\%) pancreatic, 33 $(7.7 \%)$ small intestine, 19 (4.4\%) oesophageal, and 7 (1.6\%) gall bladder cancer.

\section{Any digestive SPN}

Overall, survivors of childhood cancer were more than three times more likely to develop any SPN of the digestive system compared to that expected from the general population (SIR=3.4; 95\% Cl: 3.1-3.8), with 24 additional cases of subsequent digestive cancer observed per 100,000 person-years (Table 2). The SIR decreased significantly with increasing attained age $\left(P_{\text {trend }}<0.001\right)$, but even at age 40-49 and age 50+ years it was still 3.7-fold and 1.6-fold that expected, respectively (Table 3). In contrast, the AER increased significantly with attained age, reaching up to 79 excess SPNs per 100,000 person-years after age 50 years $\left(P_{\text {trend }}<0.001\right)$. These patterns in SIRs and AERs with attained age were confirmed in multivariable analyses (eTable 3). No significant variation in RRs and RERs with era of diagnosis was observed after adjustment for attained age in multivariable analyses (Multivariable $P_{\text {trend }} \geq 0.30$ ) (Table $3 \&$ eTable 3 ). With regard to the risk by childhood cancer type, WT and HL survivors were at greatest risk of developing a digestive SPN compared to expected ( $\mathrm{SIR}=12.1 ; 95 \% \mathrm{Cl}$ : 9.6-15.1 and $\mathrm{SIR}=7.3 ; 95 \% \mathrm{Cl}$ : 5.9-9.0, respectively) (Table 3). Although to a lesser extent, each other specific type of childhood cancer-except bone sarcoma-was also at a significantly increased risk of developing a digestive SPN. Of all childhood cancer types, the cumulative incidence for WT survivors increased the most steeply with increasing attained age; by age 30 years $0.4 \%(95 \% \mathrm{Cl}: 0.2$, 0.6 ) had developed a digestive SPN, but by age 50 years this percentage was already $4.2 \%$ $(95 \% \mathrm{Cl}: 3.1,5.6), 7.4 \%(895 \% \mathrm{Cl}: 5.5,9.8)$ by age 55 , and reached $9.6 \%(95 \% \mathrm{Cl}: 6.7,13.2)$ by age 60 years (Figure 1 ).

\section{Colorectal SPN}

Survivors experienced three times the number of colorectal cancers compared to that 
expected from the general population ( $\mathrm{SIR}=3.0 ; 95 \% \mathrm{Cl}: 2.6-3.4)$, with 11 excess colorectal cancers per 100,000 person-years (Table 2). The SIR was significantly greater at younger ages at diagnosis $\left(P_{\text {trend }}<0.001\right)$ (Table 3$)$-this trend was confirmed in multivariable analyses $\left(P_{\text {trend }}=0.001\right)$ (eTable 3$)$. Overall, the SIR of developing a colon SPN $(S I R=3.4$, $95 \% \mathrm{Cl}: 2.9,4.1)$ was greater than that of developing a rectum SPN $(\mathrm{SIR}=2.3,95 \% \mathrm{Cl}: 1.8$, 2.9). In multivariable analyses there was no significant association with era of childhood cancer diagnosis after adjustment for confounders, particularly attained age, for both SIR and AER $\left(P_{\text {trend }} \geq 0.77\right)$ (Table 3 \& eTable 3$)$. The AER increased significantly with attained age, reaching 29 excess colorectal SPNs per 100,000 person-years among those aged at least 50 years. Although the AER increased with attained age, $20 \%$ of all colorectal SPNs still occurred before age 30 years. Compared to the general population, WT and HL survivors were at greatest risk of developing a colorectal SPN; both 7-times that expected. By age 55 years, $2.3 \%(95 \% \mathrm{Cl}: 1.4,3.9)$ of WT survivors and $2.3 \%(95 \% \mathrm{Cl}: 1.6,3.2)$ of $\mathrm{HL}$ survivors had developed a colorectal SPN, similar to the $2.3 \%$ experienced by members of the general population with at least 2 first-degree relatives affected with colorectal cancer (Figure 2). Soft-tissue sarcoma survivors were at similar risk of developing a colorectal SPN as members of the general population with at least one first-degree relative affected (cumulative incidence $=1.3 \%, 95 \% \mathrm{Cl}: 0.7,2.1$ ) by age 55 years.

\section{Liver SPN}

The overall SIR of developing a liver SPN was 8-fold that expected with an AER that was 4 per 100,000 person-years, but the AER increased significantly with increasing attained age from only 1 under age 20 years to 15 among those aged at least 50 years (Table 3). Survivors of WT had the highest risk of developing a liver SPN; 41-fold that expected (95\% Cl: 26.2-64.3), although most other types of childhood cancer also exhibited high SIRs (Table 3).

\section{Stomach SPN}

Overall, survivors had 3-times higher risk of stomach cancer than expected (SIR=2.9, $95 \% \mathrm{Cl}: 2.1-3.9)$, with 2 excess cases per 100,000 person-years in excess of that expected (Table 3). Survivors diagnosed in more recent calendar years appeared to be at higher risk of developing a stomach SPN than those diagnosed before $1970\left(P_{\text {trend }}=0.04\right)($ eTable 3). With regard to childhood cancer type, survivors of WT and HL were at greatest risk relative to the general population with SIRs that were nearly 9-fold. 


\section{Pancreas SPN}

Overall, survivors experienced 3-fold the number of pancreatic SPNs (SIR=3.2; 95\%Cl: $2.3-$ 4.2), with 2 excess cases per 100,000 person-years compared to that expected from the general population (Table 3). The SIR decreased significantly with increasing attained age, but even beyond age 40 years survivors were still at over 2 -fold risk compared to that expected ( $\mathrm{SIR}=2.3,95 \% \mathrm{Cl}: 1.6-3.4)$. The risk of developing a pancreatic SPN was greatest for survivors of WT and HL at 17-fold and 7-fold compared to that expected, respectively.

Small intestine, oesophagus, and gall bladder SPNs

The highest SIR of any digestive SPN was observed for SPNs of the small intestine $(\mathrm{SIR}=11.3,95 \% \mathrm{Cl}: 7.8,15.9)$ although the AER was relatively modest $(\mathrm{AER}=2)$ when, for example, compared to the AER of colorectal SPNs (AER=11) (Table 2). The SIR for oesophagus SPNs was the lowest of all digestive SPNs (SIR=1.7, 95\% CI: 1.0, 2.7). The SIR for gall bladder SPNs was non-significant $(\mathrm{SIR}=2.4,95 \% \mathrm{Cl}: 0.9,4.9)$, but only $7 \mathrm{SPN}$ were observed at this site. 


\section{Discussion}

\section{Main findings}

In this by far the largest-ever cohort study investigating the risks of digestive SPNs in survivors of childhood cancer we provide, for the first time, accurate risk estimates of specific digestive cancers such as colorectal, liver, pancreas and stomach after age 40 years. Important findings include that for WT and HL survivors the cumulative incidence of developing colorectal cancer exceeds $2 \%$ by age 55 and is similar to that observed among individuals from the general population with at least 2 first-degree relatives affected by colorectal cancer. Other novel findings include the identification of a substantial risk of developing any digestive SPN among WT survivors in that by age 60 the cumulative risk is nearly $10 \%$ where only $1.4 \%$ was expected.

\section{Previous studies}

When evaluating the risk of developing any digestive SPNs among all childhood cancer survivors combined, the 3.4-fold SIR observed in this study was consistent with SIRs from all $[3,4,7,11]$ but one previous study [30]. For colorectal cancer specifically, the SIRs observed in the current study were generally also consistent with previous studies [3,7,9-11,30-32]. Although previous studies had fewer person-years beyond age 40, SIRs generally also decreased and AERs increased with increasing attained age or follow-up [3,30]. The identified risks of developing colorectal SPNs, particularly among WT and HL survivors, are coherent with a preponderant role of radiation exposure of the abdominal area as demonstrated in smaller studies or studies with shorter follow-up $[3,7,8,32]$. The increased risk of developing a colorectal SPN among STS survivors which is similar to the risk of individuals from the general population with at least 1 first degree relative has not been reported before, but is likely related to abdominal radiation exposure as well as the majority of STSs occurred in the abdomino-pelvic area (62\%). The risks reported here are likely to be an underestimate of the actual risk among those treated with abdominal radiotherapy as not all WT, HL and STS survivors included in this study will have been treated with radiotherapy to the abdominal area. Although exposure of the abdomen to radiation is likely to be the principal risk factor, previous studies among childhood cancer and young HL survivors have also demonstrated that exposure to procarbazine and/or cisplatin increases the risk of subsequent primary colorectal cancer [7,32].

For liver, stomach, and pancreatic SPNs the overall SIRs were all significantly elevated, but most previous studies had very few observed numbers of such SPNs complicating comparisons of the risks observed between studies $[10,11,30,33]$.To our knowledge, no previous study has had sufficient statistical power to satisfactorily investigate 
the risks of these SPNs by type of childhood cancer. In this study, particularly survivors of WT were at substantial risk of developing liver SPNs compared to the general population with a 41-fold increased SIR. Although investigation of treatment related factors was not feasible here, this substantially increased risk is likely attributable to radiation given for the original WT as the liver would most likely have been in the radiation field. Whilst the AER of developing stomach cancer was low, very high SIRs (9-fold expected) of stomach cancer were observed for survivors of a previous WT and HL. Previous studies among HL survivors demonstrated a dose-response with cumulative radiation dose received by the stomach and risk of developing stomach cancer [34,35], but to our knowledge no study suggested that survivors of a WT were also at substantially increased risk. With regard to pancreatic cancer, particularly WT survivors were at a very high risk relative to the general population (17-fold increased SIR) and to our knowledge no previous study reported risks for SPNs of the pancreas among WT survivors.

\section{Risk by era of diagnosis}

After adjustment for potential confounders in multivariable analyses, particularly with regard to attained age, no significant variations in relative or absolute risks were detected by era of diagnosis, except for the RR of stomach SPNs. It is important to note that survivors treated more recently have not yet reached ages at which in the general population the risks of developing digestive neoplasm increases substantially (i.e. beyond age 40 years) and thus any inferences with regard to era of diagnosis in relation to the long-term risk of developing a digestive SPN are difficult to make. Further follow-up of more recently treated survivors will be required to address such questions in the future.

\section{Clinical implications}

Firstly, current North American guidelines for the follow-up of survivors of childhood cancer from the Children's Oncology Group suggest any survivor treated with abdominal pelvic and/or spinal radiation or total body irradiation should receive colonoscopy surveillance for colorectal cancer 5 years after treatment or at age 30 years, whichever occurs last [36]. Multitarget stool DNA testing could be considered as an alternative if survivors are not willing to undergo colonoscopy. However, these North American guidelines are largely based on expert consensus and common European guidelines specifically relating to colorectal cancer surveillance do currently not exist. Within the International Guideline Harmonisation Group (www.ighg.org) for surveillance of late effects in childhood cancer survivors, the development of an international guideline on surveillance of colorectal cancer is a priority [37]. The current results should aid in the development of such guidelines in that we provide evidence that the risk of developing colorectal cancer increases substantially from age 30 
years onwards among survivors of WT and HL, in that by age 55 years at least $2 \%$ of WT and HL survivors had developed a colorectal SPN. Given that this risk exceeds that of individuals from the general population with at least 2 first-degree relatives affected-and for whom in many countries colorectal cancer screening and surveillance guidelines recommend offering colonoscopy before age 55 years [38-40]—serious consideration should be given for implementation of colorectal cancer surveillance among WT and HL survivors.

Secondly, among WT survivors the risk of developing any digestive SPN was $7.4 \%$ by age 55 and $10 \%$ by age 60 years compared to $1 \%$ expected by age 60 . The SPNs accounting for most of this excess risk, in addition to colorectal cancer, were of liver and pancreas. Evaluating the potential benefits, harms and cost-effectiveness of surveillance options to detect such liver and pancreatic lesions early would be prudent. For example, surveillance for hepatocellular carcinoma through regular ultra-sound examinations is effective for cirrhosis and hepatitis-B patients in the general population [41]. Whether similar surveillance would be effective among WT survivors is, however, unknown and would need in-depth evaluation of its benefits, harms and costs before being implemented.

\section{Study limitations}

For the development of international guidelines, information on dose thresholds for performing surveillance would be desirable since it currently remains uncertain whether survivors treated with lower doses of cumulative radiation to the abdomen or those who received total body irradiation would also be at risk and therefore would benefit from colonoscopy surveillance or not. The influence of alkylating agents and cisplatin dose exposure on the risk of developing colorectal and other digestive SPNs also requires more investigation. However, collection of such detailed past treatment exposure for the purpose of a cohort study would require obtaining and reviewing medical records and radiotherapy treatment charts of all individuals in the entire cohort of nearly 70,000 survivors, which was practically not feasible. We are currently in the process of conducting a case-control study nested within the current cohort and should be able to address the risks of developing colorectal SPNs at the lower end of the radiation dose spectrum and influence of different types of chemotherapy exposures. In addition, we will use the relative risk estimates from the nested case-control study, together with general population rates of specific digestive cancers, to develop absolute risk prediction models for specific digestive SPNs for the survivors and thus identify particularly high risk subgroups for whom interventions (e.g. colonoscopy surveillance) are more likely to be beneficial and cost-effective. 


\section{Conclusions}

In conclusion, most childhood cancer survivor groups are at risk of developing a digestive SPN, but particularly WT and HL survivors. The risk of developing colorectal cancer among WT and HL survivors is similar to individuals from the general population with at least 2 firstdegree relatives affected. In many European countries colonoscopy surveillance before age 55 years is recommended with such family history, but not for WT and HL survivors despite a comparable risk profile. Clinically, serious consideration should be given to the implementation of colonoscopy surveillance whilst further evaluation of its benefits, harms and cost-effectiveness in WT and HL survivors is undertaken. 


\section{References}

1 Gatta G, Botta L, Rossi S, et al. Childhood cancer survival in Europe 1999-2007: results of EUROCARE-5--a population-based study. Lancet Oncol 2014;15:35-47. doi:10.1016/S1470-2045(13)70548-5

2 Olsen JH, Möller T, Anderson H, et al. Lifelong cancer incidence in 47,697 patients treated for childhood cancer in the Nordic countries. J Natl Cancer Inst 2009;101:80613. doi:10.1093/jnci/djp104

3 Reulen RC, Frobisher C, Winter DL, et al. Long-term risks of subsequent primary neoplasms among survivors of childhood cancer. JAMA 2011;305:2311-9. doi:10.1001/jama.2011.747

4 Teepen JC, van Leeuwen FE, Tissing WJ, et al. Long-Term Risk of Subsequent Malignant Neoplasms After Treatment of Childhood Cancer in the DCOG LATER Study Cohort: Role of Chemotherapy. J Clin Oncol 2017;35:2288-98. doi:10.1200/JCO.2016.71.6902

5 Turcotte LM, Liu Q, Yasui Y, et al. Temporal Trends in Treatment and Subsequent Neoplasm Risk Among 5-Year Survivors of Childhood Cancer, 1970-2015. JAMA 2017;317:814-24. doi:10.1001/jama.2017.0693

6 Cancer Research UK. Cancer incidence for common cancers, 2015. http://www.cancerresearchuk.org/health-professional/cancerstatistics/incidence/common-cancers-compared\#heading-Zero (accessed 14 Sep 2015).

7 Henderson TO, Oeffinger KC, Whitton J, et al. Secondary gastrointestinal cancer in childhood cancer survivors: a cohort study. Ann Intern Med 2012;156:757-66, W-260. doi:10.7326/0003-4819-156-11-201206050-00002

8 Nottage K, McFarlane J, Krasin MJ, et al. Secondary colorectal carcinoma after childhood cancer. J Clin Oncol 2012;30:2552-8. doi:10.1200/JCO.2011.37.8760

9 Allodji RS, Haddy N, Vu-Bezin G, et al. Risk of subsequent colorectal cancers after a solid tumor in childhood: Effects of radiation therapy and chemotherapy. Pediatr Blood Cancer 2019;66:e27495. doi:10.1002/pbc.27495

10 Inskip PD, Curtis RE. New malignancies following childhood cancer in the United States, 1973-2002. Int J Cancer 2007;121:2233-40. doi:10.1002/ijc.22827

11 Maule M, Scélo G, Pastore G, et al. Second malignancies after childhood noncentral nervous system solid cancer: Results from 13 cancer registries. Int $J$ Cancer 2011;129:1940-52. doi:10.1002/ijc.26135

12 Hjorth L, Haupt R, Skinner R, et al. Survivorship after childhood cancer: PanCare: a European Network to promote optimal long-term care. Eur J Cancer 2015;51:1203-11. doi:10.1016/j.ejca.2015.04.002 
13 Byrne J, Alessi D, Allodji RS, et al. The PanCareSurFup consortium: research and guidelines to improve lives for survivors of childhood cancer. Eur J Cancer 2018;103:238-48. doi:10.1016/j.ejca.2018.08.017

14 Grabow D, Kaiser M, Hjorth L, et al. The PanCareSurFup cohort of 83,333 five-year survivors of childhood cancer: a cohort from 12 European countries. Eur J Epidemiol 2018;33:335-49. doi:10.1007/s10654-018-0370-3

15 Bright CJ, Hawkins MM, Winter DL, et al. Risk of Soft-Tissue Sarcoma Among 69460 Five-Year Survivors of Childhood Cancer in Europe. J Natl Cancer Inst 2018;110:64960. doi:10.1093/jnci/djx235

16 Fidler MM, Reulen RC, Winter DL, et al. Risk of Subsequent Bone Cancers Among 69460 Five-Year Survivors of Childhood and Adolescent Cancer in Europe. J Natl Cancer Inst 2018;110. doi:10.1093/jnci/djx165

17 ARC/IACR Tools for Cancer Registries. Check and conversion programs for cancer registries (I). In. IARC Technical Report No. 42. Lyon; 2005, 1-40.

18 Steliarova-Foucher E, Stiller C, Lacour B, et al. International Classification of Childhood Cancer, third edition. Cancer 2005;103:1457-67. doi:10.1002/cncr.20910

19 Breslow N, Day N. Statistical Methods in Cancer Research: Volume II. The Design and Analysis of Cohort Studies. Lyon, France: : ARC Scientific Publications 1987.

20 Steliarova-Foucher E, O'Callaghan M, Ferlay J, et al. The European Cancer Observatory: A new data resource. Eur J Cancer 2015;51:1131-43. doi:10.1016/j.ejca.2014.01.027

21 International Agency for Research on Cancer. CI5plus: Cancer incidence in five continents time trends. 2016.http://ci5.iarc.fr/CI5plus/Default.aspx

22 Ferlay J, Autier P, Boniol M, et al. Estimates of the cancer incidence and mortality in Europe in 2006. Ann Oncol 2007;18:581-92. doi:10.1093/annonc/mdl498

23 Dickman PW, Sloggett A, Hills M, et al. Regression models for relative survival. Stat Med 2004;23:51-64. doi:10.1002/sim.1597

24 Hilbe JM. Negative Binomial Regression. 2nd ed. Cambridge: : Cambridge University Press 2011. doi:10.1017/CBO9780511973420

25 Gooley TA, Leisenring W, Crowley J, et al. Estimation of failure probabilities in the presence of competing risks: new representations of old estimators. Stat Med 1999;18:695-706. doi:10.1002/(sici)1097-0258(19990330)18:6<695::aid$\operatorname{sim} 60>3.0 . \operatorname{co} ; 2-0$

26 Coviello V, Bogess M. cumulative incidence estimation in the presence of competing risks. The Stata Journal 2004;4:103-12.

27 Thernau TM GP. Modeling survival data: extending the Cox model. New York: : Springer-Verlag 2000. 
28 Butterworth AS, Higgins JPT, Pharoah P. Relative and absolute risk of colorectal cancer for individuals with a family history: a meta-analysis. Eur J Cancer 2006;42:216-27. doi:10.1016/j.ejca.2005.09.023

29 Baglietto L, Jenkins MA, Severi G, et al. Measures of familial aggregation depend on definition of family history: meta-analysis for colorectal cancer. $J$ Clin Epidemiol 2006;59:114-24. doi:10.1016/j.jclinepi.2005.07.018

30 Tukenova M, Diallo I, Anderson $\mathrm{H}$, et al. Second malignant neoplasms in digestive organs after childhood cancer: a cohort-nested case-control study.

INTJRADIATONCOLBIOLPHYS 2012;82:E383-90. doi:10.1016/j.ijrobp.2011.05.069

31 Teepen JC, Kok JL, Leeuwen FE van, et al. Colorectal adenomas and cancers after childhood cancer treatment: A DCOG-LATER record linkage study. JNCI 2018;110:758-67. doi:10.1093/jnci/djx266

32 van Eggermond AM, Schaapveld M, Janus CP, et al. Infradiaphragmatic irradiation and high procarbazine doses increase colorectal cancer risk in Hodgkin lymphoma survivors. British Journal of Cancer 2017;117:306-14. doi:10.1038/bjc.2017.177

33 Olsen JH, Garwicz S, Hertz H, et al. Second malignant neoplasms after cancer in childhood or adolescence. Nordic Society of Paediatric Haematology and Oncology Association of the Nordic Cancer Registries. BMJ 1993;307:1030-6. doi:10.1136/bmj.307.6911.1030

34 Morton LM, Dores GM, Curtis RE, et al. Stomach cancer risk after treatment for hodgkin lymphoma. J Clin Oncol 2013;31:3369-77. doi:10.1200/JCO.2013.50.6832

35 Gilbert ES, Curtis RE, Hauptmann M, et al. Stomach Cancer Following Hodgkin Lymphoma, Testicular Cancer and Cervical Cancer: A Pooled Analysis of Three International Studies with a Focus on Radiation Effects. Radiat Res 2017;187:186-95. doi:10.1667/RR14453.1

36 Children's Oncology Group (COG). Long-term follow-up guidelines for survivors of childhood, adolescent, and young adult cancers, version 5.0. 2018. http://www.survivorshipguidelines.org/pdf/2018/COG_LTFU_Guidelines_v5.pdf

37 Kremer LCM, Mulder RL, Oeffinger KC, et al. A worldwide collaboration to harmonize guidelines for the long-term follow-up of childhood and young adult cancer survivors: a report from the International Late Effects of Childhood Cancer Guideline Harmonization Group. Pediatr Blood Cancer 2013;60:543-9. doi:10.1002/pbc.24445

38 Cairns SR, Scholefield JH, Steele RJ, et al. Guidelines for colorectal cancer screening and surveillance in moderate and high risk groups (update from 2002). Gut 2010;59:66689. doi:10.1136/gut.2009.179804

39 Rex DK, Boland CR, Dominitz JA, et al. Colorectal Cancer Screening: Recommendations for Physicians and Patients from the U.S. Multi-Society Task Force on Colorectal Cancer. Am J Gastroenterol 2017;112:1016-30. doi:10.1038/ajg.2017.174

40 Nederlandse Vereniging van Maag-, Darm- en Leverartsen. Nederlandse richtlijn coloscopie surveillance, 2013. 
41 Harris PS, Hansen RM, Gray ME, et al. Hepatocellular carcinoma surveillance: An evidence-based approach. World J Gastroenterol 2019;25:1550-9.

doi:10.3748/wjg.v25.i13.1550 


\section{Competing Interests}

There are no competing interests for any author.

\section{Funding}

The PanCareSurFup consortium and related work was supported by the European Union's Seventh Framework Programme for research, technological development and demonstration under grant agreement no. 257505. Additional financial support was received from: The Fondation Force de recherche sur le cancer de l'enfant (FORCE), The Italian Association for Cancer Research and the Compagnia San Paolo; The Fondo Chiara Rama ONLUS; The Swedish Childhood Cancer Fund; the French Association for Cancer Research (ARC); The French National Agency For Research (ANR) (Hope-Epi project); the French National Cancer Institute (INCA); Pfizer Foundation for Children and Adolescent Health; Slovenian Research Agency; the Swiss Paediatric Oncology Group; The Swiss Cancer League (KLS-3412-02-2014, KLS-3886-02-2016); The Swiss Cancer Research foundation (KFS-02783-02-2011, KFS-4157-02-2017); The Swiss National Science Foundation (PDFMP3_141775), The Dutch Cancer Society, The Norwegian Childhood Cancer Foundation, and Children with Cancer UK (grant no: 20457).

\section{Acknowledgements}

We are very grateful to the childhood cancer survivors whose information was used for PanCareSurFup. We also would like to thank the following individuals from each country for their contribution to data preparation: France: Angela Jackson, Florent Dayet, Amar Kahlouche, Fara Diop, Sylvie Challeton, Martine Labbé, Isao Kobayashi. Italy: Maura Massimino, Silvia Caruso, Monica Muraca, Vera Morsellino, Claudia Casella, Lucia Miligi, Anita Andreano, Andrea Biondi and the AIRTUM working group (see appendix). The Netherlands: Dutch Childhood Oncology Group LATER; Wim Tissing, Flora van Leeuwen, Marry van den Heuvel-Eibrink, Eline van Dulmen, Jacqueline Loonen, Dorine Bresters, Birgitta Versluys. Slovenia: Tina Žagar. Sweden: Ingemar Andersson, Susanne Nordenfelt. Switzerland: Elisabeth Kiraly, Gisela Michel, Vera Mitter, Shelagh Redmond and the Swiss Paediatric Oncology Group (www.spog.ch).UK: Julie Kelly.

The views expressed in this publication are those of the authors and do not necessarily represent those of the funders or collaborating institutions. 
Table 1. Characteristics of all 69,460 five-year survivors in the PanCareSurFup study and number of subsequent primary neoplasm of the digestive system.

\begin{tabular}{|c|c|c|c|}
\hline & & $\mathbf{N}(\%)$ & digestive SPNa \\
\hline all survivors & & $69460(100 \%)$ & $427(100 \%)$ \\
\hline \multirow[t]{2}{*}{$\operatorname{sex}$} & male & $37738(54.3 \%)$ & $274(64.2 \%)$ \\
\hline & female & $31722(45.7 \%)$ & $153(35.8 \%)$ \\
\hline childhood & leukaemia & $16595(23.9 \%)$ & $30(7.0 \%)$ \\
\hline \multirow[t]{12}{*}{ cancer type } & Hodgkin's lymphoma & $6000(8.6 \%)$ & $85(19.9 \%)$ \\
\hline & non-Hodgkin's lymphoma & $3350(4.8 \%)$ & $26(6.1 \%)$ \\
\hline & central nervous system tumour & $14529(20.9 \%)$ & $60(14.1 \%)$ \\
\hline & neuroblastoma & $3169(4.6 \%)$ & $10(2.3 \%)$ \\
\hline & retinoblastoma & $2578(3.7 \%)$ & $13(3.0 \%)$ \\
\hline & Wilms' tumour & $4756(6.8 \%)$ & $77(18.0 \%)$ \\
\hline & bone tumour & $3147(4.5 \%)$ & $14(3.3 \%)$ \\
\hline & soft-tissue Sarcoma & $4501(6.5 \%)$ & $43(10.1 \%)$ \\
\hline & gonadal tumour testis & $1664(2.4 \%)$ & $10(2.3 \%)$ \\
\hline & gonadal tumours ovary & $1038(1.5 \%)$ & $17(4.0 \%)$ \\
\hline & otherb & $7770(11.2 \%)$ & $35(8.2 \%)$ \\
\hline & unclassified & $363(0.5 \%)$ & $7(1.6 \%)$ \\
\hline age at childhood & $0-4$ & $22013(31.7 \%)$ & $117(27.3 \%)$ \\
\hline cancer diagnosis & $5-9$ & $14846(21.4 \%)$ & $84(19.6 \%)$ \\
\hline \multirow[t]{2}{*}{ (years) } & $10-14$ & $11199(16.1 \%)$ & $80(18.7 \%)$ \\
\hline & $15-19$ & $21402(30.8 \%)$ & $147(34.3 \%)$ \\
\hline data provider & France & $3138(4.5 \%)$ & $63(14.8 \%)$ \\
\hline \multirow[t]{12}{*}{ country } & Hungary & $4885(7.0 \%)$ & $17(4.0 \%)$ \\
\hline & Italy (population-based)c & $7476(10.8 \%)$ & $14(3.3 \%)$ \\
\hline & Italy (hospital-based)d & $1490(2.1 \%)$ & $4(0.9 \%)$ \\
\hline & Netherlands & $6044(8.7 \%)$ & $17(4.0 \%)$ \\
\hline & Denmark & $4840(7.0 \%)$ & $31(7.3 \%)$ \\
\hline & Sweden & $7709(11.1 \%)$ & $14(3.3 \%)$ \\
\hline & Norway & $3783(5.4 \%)$ & $11(2.6 \%)$ \\
\hline & Finland & $6229(9.0 \%)$ & $40(9.4 \%)$ \\
\hline & Iceland & $275(0.4 \%)$ & $0(0.0 \%)$ \\
\hline & Slovenia & $1252(1.8 \%)$ & $9(2.1 \%)$ \\
\hline & Switzerland & $4379(6.3 \%)$ & $5(1.2 \%)$ \\
\hline & United Kingdom & $17960(25.9 \%)$ & $202(47.3 \%)$ \\
\hline era of & $<1970$ & $8993(12.9 \%)$ & $178(41.7 \%)$ \\
\hline \multirow[t]{3}{*}{ diagnosis } & $1970-79$ & $13479(19.4 \%)$ & $152(35.6 \%)$ \\
\hline & $1980-89$ & $20900(30.1 \%)$ & $78(18.3 \%)$ \\
\hline & $1990-2008$ & $26088(37.6 \%)$ & $19(4.4 \%)$ \\
\hline attained & $<20$ & $15405(22.2 \%)$ & $24(5.6 \%)$ \\
\hline \multirow[t]{4}{*}{ age (years) } & $20-29$ & $18877(27.2 \%)$ & $66(15.5 \%)$ \\
\hline & $30-39$ & $17145(24.7 \%)$ & $107(25.1 \%)$ \\
\hline & $40-49$ & $10969(15.8 \%)$ & $124(29.0 \%)$ \\
\hline & $50+$ & $7064(10.2 \%)$ & $106(24.8 \%)$ \\
\hline
\end{tabular}

aA total of 427 digestive SPNs were observed among 413 survivors.

b"Other" category includes; malignant melanomas $(\mathrm{N}=1390)$, thyroid carcinomas $(\mathrm{N}=1229)$, other and unspecified carcinomas $(\mathrm{N}=1111), \mathrm{Burkitt}$ lymphoma ( $\mathrm{N}=680)$, malignant extracranial/extragonadal germ cell tumours $(\mathrm{N}=421)$, skin carcinomas $(\mathrm{N}=386)$, unspecified lymphomas $(\mathrm{N}=377)$, hepatoblastoma $(\mathrm{N}=314)$, miscellaneous lymphoreticular neoplasms $(\mathrm{N}=274)$, nasopharyngeal carcinomas $(\mathrm{N}=169)$, renal carcinomas $(\mathrm{N}=121)$, other peripheral nervous cell tumours $(\mathrm{N}=91)$, hepatic carcinomas $(\mathrm{N}=81)$, adrenocortical carcinomas $(\mathrm{N}=70)$, other specified malignant tumours $(\mathrm{N}=50)$, unspecified malignant renal tumours $(\mathrm{N}=35)$, unspecified malignant hepatic tumours $(\mathrm{N}=15)$, other and unspecified malignant tumours ( $\mathrm{N}=956)$.

cPopulation-based cohort from the childhood cancer registry of Piedmont region in Italy.

dHospital-based registry from the Italian Registry of Off Therapy Patients, Genova. 
Table 2. Standardised incidence ratios and absolute excess risks for specific digestive subsequent primary neoplasms (SPNs)

\begin{tabular}{lcccc}
\hline SPN & Obs & Exp $^{\mathbf{a}}$ & SIR $(95 \% \mathbf{C l})$ & AER $(\mathbf{9 5} \% \mathbf{C I})^{\mathbf{b}}$ \\
\hline any digestive & 427 & 124.5 & $3.4(3.1,3.8)$ & $24(21,27)$ \\
colorectal & 214 & 72.5 & $3.0(2.6,3.4)$ & $11(9,13)$ \\
一 colon only & 144 & 41.8 & $3.4(2.9,4.1)$ & $8(7,10)$ \\
-rectum only & 70 & 30.7 & $2.3(1.8,2.9)$ & $3(2,4)$ \\
liver & 62 & 7.7 & $8.1(6.2,10.3)$ & $4(3,6)$ \\
stomach & 48 & 16.5 & $2.9(2.1,3.9)$ & $2(2,4)$ \\
pancreas & 44 & 13.6 & $3.2(2.3,4.3)$ & $2(2,3)$ \\
small intestine & 33 & 2.9 & $11.3(7.8,15.9)$ & $2(2,3)$ \\
oesophagus & 19 & 11.0 & $1.7(1.0,2.7)$ & $1(0,1)$ \\
gall bladder & 7 & 3.0 & $2.4(0.9,4.9)$ & $0(0,1)$ \\
\hline aExpected numbers do not add up to total for all digestive SPNs because general population cancer incidence \\
reference rates for small intestine, oesophagus, and gall bladder were obtained through ECO; others through Cl5. \\
bper 100,000 person-years
\end{tabular}


Table 3. Standardised incidence ratios and absolute excess risks for any digestive and colorectal (plus colon and rectum, separately) subsequent primary neoplasm

\begin{tabular}{|c|c|c|c|c|c|c|c|c|c|c|c|c|c|}
\hline \multirow[b]{2}{*}{ Factor } & \multirow[b]{2}{*}{ level } & \multicolumn{3}{|c|}{ any digestive } & \multicolumn{3}{|c|}{ colorectal } & \multicolumn{3}{|c|}{ colon only } & \multicolumn{3}{|c|}{ rectum only } \\
\hline & & 0 & SIR $(95 \% \mathrm{Cl})$ & AER $(95 \% \mathrm{Cl})$ & 0 & SIR $(95 \% \mathrm{Cl})$ & AER $(95 \% \mathrm{Cl})$ & 0 & SIR (95\%Cl) & AER $(95 \% \mathrm{Cl})$ & 0 & SIR (95\%Cl) & AER $(95 \% \mathrm{Cl})$ \\
\hline Overall & All combined & 427 & $3.4(3.1,3.8)$ & $24(21,27)$ & 214 & $3.0(2.6,3.4)$ & $11(9,13)$ & 144 & $3.4(2.9,4.1)$ & $8(7,10)$ & 70 & $2.3(1.8,2.9)$ & $3(2,4)$ \\
\hline \multirow[t]{3}{*}{ Sex } & Male & 274 & $3.7(3.3,4.2)$ & $30(25,35)$ & 134 & $3.4(2.9,4.0)$ & $14(11,18)$ & 90 & $4.1(3.4,5.1)$ & $10(8,13)$ & 45 & $2.5(1.9,3.4)$ & $4(2,7)$ \\
\hline & Female & 153 & $3.0(2.5,3.5)$ & $17(14,22)$ & 78 & $2.4(1.9,3.0)$ & $8(5,11)$ & 54 & $2.7(2.1,3.5)$ & $6(4,9)$ & 25 & $1.9(1.3,2.9)$ & $2(1,5)$ \\
\hline & $P_{\text {heterogeneity }}$ & & $0.02(0.02)$ & $<0.001(<0.001)$ & & $0.01(0.03)$ & $0.01(0.07)$ & & $0.01(0.08)$ & $0.02(0.28)$ & & $0.27(0.16)$ & $0.13(0.10)$ \\
\hline \multirow{5}{*}{$\begin{array}{l}\text { Age at } \\
\text { diagnosis } \\
\text { (yrs) }\end{array}$} & $0-4$ & 116 & $5.3(4.4,6.3)$ & $21(17,27)$ & 52 & $4.0(3.1,5.3)$ & $9(6,13)$ & 37 & $5.0(3.6,6.8)$ & $7(5,10)$ & 15 & $2.7(1.7,4.5)$ & $2(1,5)$ \\
\hline & $5-9$ & 84 & $4.6(3.7,5.7)$ & $24(18,31)$ & 46 & $4.2(3.2,5.7)$ & $13(9,18)$ & 37 & $5.9(4.3,8.2)$ & $11(7,16)$ & 9 & $2.0(1.0,3.8)$ & $2(0,6)$ \\
\hline & $10-14$ & 80 & $3.2(2.6,4.0)$ & $26(19,36)$ & 50 & $3.5(2.6,4.6)$ & $17(11,25)$ & 30 & $3.6(2.5,5.2)$ & $10(6,17)$ & 20 & $3.2(2.1,5.0)$ & $7(3,12)$ \\
\hline & $15-19$ & 147 & $2.5(2.1,2.9)$ & $26(20,34)$ & 66 & $1.9(1.5,2.5)$ & $9(6,15)$ & 40 & $2.0(1.5,2.8)$ & $6(3,11)$ & 26 & $1.8(1.2,2.6)$ & $3(1,8)$ \\
\hline & $P_{\text {trend }}$ & & $<0.001(0.01)$ & $0.24(<0.001)$ & & $<0.001(<0.001)$ & $0.44(<0.001)$ & & $<0.001(0.01)$ & $0.97(<0.001)$ & & $0.28(0.13)$ & $0.17(0.05)$ \\
\hline \multirow{4}{*}{$\begin{array}{l}\text { Era of } \\
\text { diagnosis }\end{array}$} & $1970-1979$ & 152 & $5.8(4.9,6.8)$ & $36(29,43)$ & 76 & $4.9(3.9,6.2)$ & $17(13,23)$ & 54 & $6.2(4.7,8.1)$ & $13(9,18)$ & 22 & $3.3(2.2,5.0)$ & $4(2,8)$ \\
\hline & $1980-1989$ & 78 & $6.4(5.1,8.0)$ & $16(13,21)$ & 35 & $4.7(3.4,6.5)$ & $7(5,11)$ & 23 & $5.0(3.3,7.5)$ & $5(3,8)$ & 12 & $4.2(2.4,7.3)$ & $2(1,5)$ \\
\hline & $1990-2008$ & 19 & $7.1(4.5,11.2)$ & $8(5,14)$ & 10 & $6.7(3.6,12.5)$ & $4(2,9)$ & 5 & $4.9(2.0,11.7)$ & $2(1,6)$ & 5 & $10.7(4.5,25.8)$ & $2(1,6)$ \\
\hline & $P_{\text {trend }}$ & & $<0.001(0.30)$ & $<0.001(0.53)$ & & $<0.001(0.83)$ & $<0.001(0.77)$ & & $<0.001(0.37)$ & $<0.001(0.44)$ & & $<0.001(0.05)$ & $0.23(0.06)$ \\
\hline Attained & $0-20$ & 24 & $14.8(9.9,22.1)$ & $5(4,8)$ & 16 & $18.7(11.4,30.5)$ & $4(2,6)$ & 13 & $16.5(9.6,28.3)$ & $3(2,5)$ & 3 & $44.3(14.3,137.3)$ & $1(0,2)$ \\
\hline \multirow{5}{*}{ Age (yrs) } & $20-29$ & 66 & $9.3(7.3,11.9)$ & $14(11,18)$ & 28 & $6.4(4.4,9.3)$ & $6(4,9)$ & 20 & $6.7(4.3,10.4)$ & $4(2,7)$ & 8 & $5.8(2.9,11.6)$ & $2(1,4)$ \\
\hline & $30-39$ & 107 & $6.0(5.0,7.3)$ & $34(27,43)$ & 52 & $4.9(3.7,6.4)$ & $16(11,22)$ & 35 & $5.5(3.9,7.6)$ & $11(7,16)$ & 17 & $4.0(2.5,6.5)$ & $5(3,9)$ \\
\hline & $40-49$ & 124 & $3.7(3.1,4.5)$ & $75(59,96)$ & 65 & $3.4(2.7,4.4)$ & $38(27,54)$ & 48 & $4.6(3.4,6.0)$ & $31(22,45)$ & 17 & $2.0(1.2,3.2)$ & $7(3,18)$ \\
\hline & $50+$ & 106 & $1.6(1.4,2.0)$ & $79(48,129)$ & 53 & $1.4(1.1,1.8)$ & $29(12,74)$ & 28 & $1.3(0.9,1.9)$ & $13(3,59)$ & 25 & $1.5(1.0,2.2)$ & $16(5,52)$ \\
\hline & $P_{\text {trend }^{*}}$ & & $<0.001(<0.001)$ & $<0.001(<0.001)$ & & $<0.001(<0.001)$ & $<0.001(<0.001)$ & & $<0.001(<0.001)$ & $<0.001(<0.001)$ & & $<0.001(0.03)$ & $<0.001(<0.001)$ \\
\hline \multirow{12}{*}{$\begin{array}{l}\text { Type of } \\
\text { childhood } \\
\text { cancer }\end{array}$} & leukaemia & 30 & $3.3(2.3,4.7)$ & $8(5,14)$ & 12 & $2.2(1.2,3.8)$ & $3(1,7)$ & 10 & $3.0(1.6,5.6)$ & $3(1,7)$ & 2 & $0.9(0.2,3.6)$ & 0 \\
\hline & CNS tumour & 60 & $2.0(1.5,2.5)$ & $11(7,19)$ & 36 & $2.0(1.5,2.8)$ & $7(4,13)$ & 21 & $2.1(1.3,3.2)$ & $4(2,9)$ & 15 & $2.0(1.2,3.3)$ & $3(1,8)$ \\
\hline & neuroblastoma & 10 & $3.2(1.7,5.9)$ & $11(5,27)$ & 5 & $2.7(1.1,6.5)$ & $5(1,21)$ & 3 & $2.8(0.9,8.8)$ & $3(1,18)$ & 2 & $2.5(0.6,10.2)$ & $2(0,19)$ \\
\hline & retinoblastoma & 13 & $1.8(1.0,3.1)$ & $8(2,28)$ & 7 & $1.6(0.8,3.4)$ & $4(1,26)$ & 5 & $2.0(0.9,4.9)$ & $4(1,20)$ & 2 & $1.1(0.3,4.3)$ & 0 \\
\hline & Wilms Tumour & 77 & $12.1(9.6,15.1)$ & $65(51,83)$ & 26 & $6.8(4.6,10.0)$ & $20(13,32)$ & 22 & $10.0(6.6,15.2)$ & $18(11,29)$ & 4 & $2.5(0.9,6.6)$ & $2(0,11)$ \\
\hline & bone sarcoma & 14 & $1.6(0.9,2.6)$ & $9(2,38)$ & 4 & $0.8(0.3,2.1)$ & 0 & 2 & $0.7(0.2,2.7)$ & 0 & 2 & $0.9(0.2,3.6)$ & 0 \\
\hline & soft-tissue sarcoma & 43 & $3.6(2.7,4.8)$ & $34(22,51)$ & 25 & $3.6(2.4,5.3)$ & $20(11,34)$ & 15 & $3.8(2.3,6.2)$ & $12(6,24)$ & 10 & $3.3(1.8,6.2)$ & $8(3,18)$ \\
\hline & gonadal testis & 10 & $4.2(2.3,7.8)$ & $29(13,64)$ & 4 & $3.1(1.2,8.3)$ & $10(2,43)$ & 3 & $4.2(1.3,12.9)$ & $9(2,38)$ & 1 & $1.8(0.2,12.6)$ & $2(0,148)$ \\
\hline & gonadal ovary & 17 & $4.9(3.0,7.9)$ & $63(34,114)$ & 10 & $4.6(2.5,8.5)$ & $36(16,80)$ & 5 & $3.8(1.6,9.1)$ & $17(5,56)$ & 5 & $5.8(2.4,13.9)$ & $19(7,55)$ \\
\hline & other & 35 & $1.8(1.3,2.5)$ & $11(5,24)$ & 17 & $1.5(0.9,2.4)$ & $4(1,17)$ & 11 & $1.6(0.9,3.0)$ & $3(1,14)$ & 6 & $1.3(0.6,2.8)$ & $1(0,39)$ \\
\hline & not classifiable & 7 & $2.8(1.3,5.9)$ & $38(12,120)$ & 4 & $3.3(1.3,8.9)$ & $24(6,96)$ & 3 & $4.8(1.6,15.0)$ & $20(5,84)$ & 1 & $1.7(0.2,12.2)$ & $4(0,379)$ \\
\hline & $P_{\text {heterogeneity }^{*}}$ & & $<0.001(<0.001)$ & $<0.001(<0.001)$ & & $<0.001(<0.001)$ & $<0.001(<0.001)$ & & $<0.001(<0.001)$ & $<0.001(<0.001)$ & & $0.09(0.01)$ & $0.01(0.01)$ \\
\hline
\end{tabular}

* $P$-values in brackets refer to P-value derived from multivariable Poisson regression; $0=$ observed number 
Table 3 (continued). Standardised incidence ratios and absolute excess risks for subsequent liver, stomach and pancreas cancer.

\begin{tabular}{|c|c|c|c|c|c|c|c|c|c|c|}
\hline \multirow[b]{2}{*}{ Factor } & \multirow[b]{2}{*}{ level } & \multicolumn{3}{|c|}{ liver } & \multicolumn{3}{|c|}{ stomach } & \multicolumn{3}{|c|}{ pancreas } \\
\hline & & 0 & SIR $(95 \% \mathrm{Cl})$ & AER $(95 \% \mathrm{Cl})$ & 0 & SIR (95\%Cl) & AER $(95 \% \mathrm{Cl})$ & 0 & SIR $(95 \% \mathrm{Cl})$ & AER $(95 \% \mathrm{Cl})$ \\
\hline Overall & All combined & 62 & $8.1(6.2,10.3)$ & $4(3,6)$ & 48 & $2.9(2.1,3.9)$ & $2(2,4)$ & 44 & $3.2(2.3,4.2)$ & $2(2,3)$ \\
\hline \multirow[t]{3}{*}{ Sex } & Male & 38 & $7.3(5.3,1)$ & $5(3,7)$ & 30 & $2.9(2.0,4.2)$ & $3(2,5)$ & 27 & $3.5(2.4,5.1)$ & $3(2,5)$ \\
\hline & Female & 24 & $9.7(6.5,14.4)$ & $4(2,6)$ & 18 & $2.9(1.8,4.6)$ & $2(1,4)$ & 17 & $2.9(1.8,4.6)$ & $2(1,4)$ \\
\hline & $P_{\text {heterogeneity }}$ & & $0.29(0.77)$ & $0.33(0.14)$ & & $0.96(0.77)$ & $0.40(0.62)$ & & $0.54(0.81)$ & $0.36(0.24)$ \\
\hline \multirow{5}{*}{$\begin{array}{l}\text { Age at } \\
\text { diagnosis } \\
\text { (yrs) }\end{array}$} & $0-4$ & 22 & $14.1(9.3,21.4)$ & $5(3,7)$ & 9 & $3.3(1.7,6.4)$ & $1(1,4)$ & 12 & $5.5(3.1,9.7)$ & $2(1,4)$ \\
\hline & $5-9$ & 14 & $11.3(6.7,19.1)$ & $5(3,8)$ & 4 & $1.7(0.6,4.6)$ & $1(0,6)$ & 7 & $3.8(1.8,8.0)$ & $2(1,5)$ \\
\hline & $10-14$ & 7 & $4.6(2.2,9.6)$ & $3(1,7)$ & 8 & $2.5(1.3,5.0)$ & $2(1,7)$ & 9 & $3.4(1.8,6.5)$ & $3(1,8)$ \\
\hline & $15-19$ & 19 & $5.7(3.6,8.9)$ & $5(3,8)$ & 27 & $3.3(2.2,4.8)$ & $6(3,10)$ & 16 & $2.3(1.4,3.8)$ & $3(1,6)$ \\
\hline & $P_{\text {trend" }}$ & & $<0.001(0.59)$ & $0.66(0.70)$ & & $0.66(0.82)$ & $0.01(0.19)$ & & $0.02(0.34)$ & $0.64(0.62)$ \\
\hline \multirow{5}{*}{$\begin{array}{l}\text { Era of } \\
\text { diagnosis }\end{array}$} & $<1970$ & 29 & $6.2(4.3,8.9)$ & $8(5,12)$ & 17 & $1.6(1.0,2.6)$ & $2(1,7)$ & 20 & $2.0(1.3,3.1)$ & $3(1,8)$ \\
\hline & $1970-1979$ & 20 & $11.4(7.4,17.7)$ & $5(3,8)$ & 16 & $4.4(2.7,7.1)$ & $3(2,7)$ & 17 & $6.5(4.0,10.5)$ & $4(2,7)$ \\
\hline & $1980-1989$ & 9 & $9.5(4.9,18.2)$ & $2(1,4)$ & 12 & $6.6(3.7,11.6)$ & $3(1,5)$ & 6 & $6.4(2.9,14.3)$ & $1(0,3)$ \\
\hline & 1990-2008 & 4 & $14.2(5.3,37.9)$ & $2(1,5)$ & 3 & $7.1(2.3,22.0)$ & $1(0,5)$ & 1 & $5.6(0.8,39.7)$ & $0(0,4)$ \\
\hline & $P_{\text {trend }^{*}}$ & & $0.06(0.77)$ & $<0.001(0.59)$ & & $<0.001(0.04)$ & $0.44(0.46)$ & & $<0.001(0.23)$ & $0.02(0.93)$ \\
\hline Attained & $0-19$ & 5 & $13.8(5.7,33.0)$ & $1(0,3)$ & 1 & $8.7(1.2,61.8)$ & $0(0,2)$ & 1 & $9.9(1.4,70.2)$ & $0(0,2)$ \\
\hline \multirow{4}{*}{ Age (yrs) } & $20-29$ & 13 & $19.8(11.5,34.1)$ & $3(2,5)$ & 8 & $6.6(3.3,13.1)$ & $2(1,4)$ & 5 & $12.4(5.2,29.8)$ & $1(0,3)$ \\
\hline & $30-39$ & 13 & $12.6(7.3,21.6)$ & $5(3,8)$ & 15 & $4.8(2.9,7.9)$ & $5(2,9)$ & 11 & $7.8(4.3,14.1)$ & $4(2,7)$ \\
\hline & $40+$ & 31 & $5.5(3.9,7.8)$ & $15(10,23)$ & 24 & $2.0(1.3,3.0)$ & $7(3,15)$ & 27 & $2.3(1.6,3.4)$ & $9(5,17)$ \\
\hline & $P_{\text {trend }^{*}}$ & & $<0.001(0.06)$ & $<0.001(<0.001)$ & & $<0.001(0.08)$ & $<0.001(<0.001)$ & & $<0.001(0.03)$ & $<0.001(<0.001)$ \\
\hline \multirow{14}{*}{$\begin{array}{l}\text { Type of } \\
\text { childhood } \\
\text { cancer }\end{array}$} & leukaemia & 5 & $7.4(3.1,17.7)$ & $2(1,5)$ & 1 & $0.8(0.1,5.8)$ & 0 & 2 & $2.6(0.6,10.4)$ & $0(0,5)$ \\
\hline & Hodgkin lymphoma & 6 & $8.0(3.6,17.9)$ & $5(2,13)$ & 14 & $8.5(5.0,14.3)$ & $13(7,23)$ & 9 & $7.1(3.7,13.7)$ & $8(4,17)$ \\
\hline & NHL & 1 & $2.2(0.3,15.5)$ & $1(0,33)$ & 0 & 0 & 0 & 3 & $3.7(1.2,11.5)$ & $4(1,17)$ \\
\hline & CNS tumour & 10 & $5.5(3.0,10.3)$ & $3(1,7)$ & 4 & $1.0(0.4,2.7)$ & 0 & 4 & $1.2(0.4,3.2)$ & $0(0,103)$ \\
\hline & neuroblastoma & 2 & $8.7(2.2,34.8)$ & $3(1,14)$ & 1 & $2.6(0.4,18.1)$ & $1(0,25)$ & 0 & 0 & 0 \\
\hline & retinoblastoma & 2 & $4.5(1.1,18.1)$ & $2(0,13)$ & 1 & $1.2(0.2,8.2)$ & 0 & 1 & $1.3(0.2,9.2)$ & 0 \\
\hline & Wilms Tumour & 19 & $41.0(26.2,64.3)$ & $17(11,27)$ & 7 & $8.8(4.2,18.4)$ & $6(2,13)$ & 11 & $16.9(9.4,30.5)$ & $10(5,18)$ \\
\hline & bone Sarcoma & 0 & & & 8 & $6.7(3.4,13.4)$ & $12(5,27)$ & 1 & $1.0(0.1,6.8)$ & 0 \\
\hline & soft-tissue sarcoma & 7 & $9.5(4.5,19.9)$ & $7(3,16)$ & 2 & $1.3(0.3,5.1)$ & $0(0,268)$ & 4 & $2.9(1.1,7.8)$ & $3(1,13)$ \\
\hline & gonadal testis & 2 & $11.2(2.8,44.9)$ & $7(1,31)$ & 5 & $6.5(2.7,15.7)$ & $9(3,25)$ & 2 & $7.9(2.0,31.6)$ & $7(1,32)$ \\
\hline & gonadal ovary & 3 & $19.8(6.4,61.4)$ & $13(4,43)$ & 4 & $1.5(0.6,4.1)$ & $1(0,17)$ & 0 & 0 & 0 \\
\hline & other & 4 & $3.7(1.4,9.7)$ & $2(1,8)$ & 1 & $2.0(0.3,14.5)$ & $4(0,202)$ & 7 & $3.0(1.4,6.3)$ & $3(1,10)$ \\
\hline & not classifiable & 1 & $7.0(1.0,49.8)$ & $7(1,71)$ & 1 & $0.8(0.1,5.8)$ & 0 & 0 & 0 & 0 \\
\hline & $P_{\text {heterogeneity }}$ & & $0.001(<0.001)$ & $<0.001(<0.001)$ & & $<0.001(<0.001)$ & $<0.001(<0.001)$ & & $<0.001(<0.001)$ & $<0.001(<0.001)$ \\
\hline
\end{tabular}

${ }^{*} \mathrm{P}$-values in brackets refer to $\mathrm{P}$-value derived from multivariable Poisson regression; $\mathrm{O}=$ observed number 
Number at risk

All survivors

Hodgkin lymphoma

Non-Hodgkin lymphoma

CNS tumour

Wilms tumour

Soft-tissue sarcoma
20 yrs

54041

5497

2828

11139

3637

3661
30 yrs

35157

3500

1882

7192

2522

2506
40 yrs

17995

1852

945

4005

1239

1456
50 yrs

7025

661

366

1823

373

637
55 yrs

3943

340

230

1042

172

411
60 yrs

2004

158

126

546

71

223 